\title{
Editorial: Exposure to
} Endocrine-Disrupting Chemicals and Cardiometabolic Disease: A Developmental Origins Approach

\author{
Wei Perng ${ }^{1,2,3 *}$, Jaclyn M. Goodrich ${ }^{4}$, Andres Cardenas $^{5}$ and Deborah J. Watkins ${ }^{4}$ \\ 1 Lifcourse Epidemiology of Adiposity and Diabetes (LEAD) Center, University of Colorado Anschutz Medical Campus, Aurora, \\ CO, United States, ${ }^{2}$ Department of Epidemiology, Colorado School of Public Health, Aurora, CO, United States, ${ }^{3}$ Department \\ of Nutritional Sciences, University of Michigan School of Public Health, Ann Arbor, MI, United States, ${ }^{4}$ Department of \\ Environmental Health Sciences, University of Michigan School of Public Health, Ann Arbor, MI, United States, ${ }^{5}$ Division of \\ Environmental Health Sciences, School of Public Health, University of California, Berkeley, Berkeley, CA, United States
}

Keywords: Developmental Origins of Health and Disease (DOHaD), endocrine-disrupting chemical, obesity, cardio-metabolic abnormalities, children, adolescents

\section{Editorial on the Research Topic}

Exposure to Endocrine-Disrupting Chemicals and Cardiometabolic Disease: A Developmental Origins Approach

Over the latter half of the twentieth century, community-level exposure to mercury-contaminated grain in Iraq and seafood in Japan exemplified the toxic consequences of environmental pollutants on fetal brain development (1). Since these devastating historical events, researchers have uncovered detrimental effects of toxicants even at low-levels of exposure $(2,3)$. Many of these toxicants are thought to act by disrupting the normal biosynthesis, metabolism, or action of hormones in the body, and are thus referred to as Endocrine Disrupting Chemicals (EDCs).

In parallel with interest in the role of EDCs in disease etiology, a trio of studies published in the Lancet in the late 1980s/early 1990s put forth the hypothesis that environmental exposures during vulnerable developmental stages-the in utero period in particular-have a permanent impact on an organism's metabolic phenotype (4-6). What was initially referred to as "Barker's hypothesis" subsequently expanded into the "Developmental Origins of Health and Disease (DOHaD) hypothesis" which posits that environmental exposures during multiple sensitive periods of development (not just the in utero period) have a lasting impact on health and disease risk (7).

This Research Topic places the fields of environmental epidemiology and exposure assessment within the $\mathrm{DOHaD}$ framework. We received four articles that provide novel insights into the effect of environmental exposures during sensitive periods of development on cardiometabolic traits across childhood and adolescence:

Vafeiadi et al. examined associations of phthalate exposure during gestation and childhood $(\sim 4$ years of age) with adiposity and other cardiometabolic traits in 500 Greek children in the Rhea mother-child cohort. While the authors did not find any consistent associations of prenatal phthalate exposure with adiposity or metabolic risk at age 4 years, they detected an inverse association between childhood exposure to certain phthalate metabolites-namely, MEP, MnBP, $\mathrm{MBzP}$ - with blood pressure, and a positive association of MiBP with cholesterol; as well as sex-specific associations of concurrent phthalate exposure with adiposity indicators.

Using a similar study design, Moynihan et al. investigated associations of prenatal and concurrent cadmium exposure on adiposity at age 8-14 years among 185 participants in the Early 
Life Exposure in Mexico to ENvironmental Toxicants (ELEMENT) Project. The authors reported an inverse relationship between prenatal cadmium exposure and adiposity during late childhood/early adolescence-an association that was driven by females.

In another study utilizing the ELEMENT cohort, Bowman et al. explored associations between trimester-specific and adolescent phthalate exposure with adiposity at two time-points across the pubertal transition among 109 boys and 114 boys, and assessed DNA methylation as a potential mediator to these relationships. Exposure to certain phthalates (MBP and MiBP) during early gestation was associated with an increase in adiposity among girls, whereas concurrent exposure to $\mathrm{MBzP}$ was associated with decreased adiposity among boys. The investigators noted differential DNA methylation of two growth-related genes (H19 and HSD11B2) in relation to prenatal and adolescent phthalate exposure, as well as with respect to adiposity across adolescence, suggesting involvement of these genes in associations of interest. Mediation analysis did not reveal any statistically significant pathways; however the analysis was likely underpowered. Future, larger studies exploring epigenetic mechanisms as a mediator between early life environmental exposures and childhood outcomes are warranted.

In a methodological study, Zhou et al. leveraged targeted metabolomics analyses in plasma and urine of 115 pregnant women at $\sim 26$ weeks gestation to identify novel metabolomics biomarkers of phthalate

\section{REFERENCES}

1. Myers GJ, Davidson PW. Does methylmercury have a role in causing developmental disabilities in children? Environ Health Perspect. (2000) 108(Suppl. 3):413-20. doi: 10.1289/ehp.00108 s3413

2. Braun JM. Early-life exposures to EDCs: role in childhood obesity and neurodevelopment. Nat Rev Endocrinol. (2017) 13:161-73. doi: 10.1038/nrendo.2016.186

3. Xu C, Shu Y, Fu Z, Hu Y, Mo X. Associations between lead concentrations and cardiovascular risk factors in U.S. adolescents. Sci Rep. (2017). 7:9121. doi: 10.1038/s41598-017-09701-4

4. Barker DJ, Osmond C. Infant mortality, childhood nutrition, and ischaemic heart disease in England and Wales. Lancet. (1986) 1:1077-81. doi: 10.1016/s0140-6736(86)91340-1

5. Barker DJ, Winter PD, Osmond C, Margetts B, Simmonds SJ. Weight in infancy and death from ischaemic heart disease. Lancet. (1989) 2:577-80. doi: 10.1016/s0140-6736(89)90710-1 exposure, and to gain insight into perturbed biochemical pathways. The authors identified metabolites in both plasma and urine that suggested the involvement of diverse pathways, including enhanced lipid, steroid, and nucleic metabolism, and an upregulated inflammatory response in relation to higher phthalate concentrations in both plasma and urine.

Beyond the work carried out by contributors to this Research Topic, future research in this field is needed to interrogate the effects of toxicant mixtures, which more accurately reflect real-life exposure than single toxicants or summary scores of multiple toxicants; the interactive effects of toxicant exposure during multiple sensitive periods throughout the life course, which reflects accumulation of risk; and studies that consider multiple mediating mechanisms, including but not limited to epigenetics, proteomics, metabolomics, and the microbiome to gain a more comprehensive understanding of mechanistic pathways that not only link environmental exposures to adverse health outcomes but also influence susceptibility to toxicity from these exposures.

\section{AUTHOR CONTRIBUTIONS}

WP conceived the idea for the editorial, wrote the initial draft, and incorporated comments from co-editors. JG, AC, and DW provided critical feedback. All authors approved the final version of the editorial.
6. Barker DJ, Gluckman PD, Godfrey KM, Harding JE, Owens JA, Robinson JS. Fetal nutrition and cardiovascular disease in adult life. Lancet. (1993) 341:938-41. doi: 10.1016/0140-6736(93)91224-a

7. Wadhwa PD, Buss C, Entringer S, Swanson JM. Developmental origins of health and disease: brief history of the approach and current focus on epigenetic mechanisms. Semin Reprod Med. (2009). 27:358-68. doi: $10.1055 /$ s-0029-1237424

Conflict of Interest: The authors declare that the research was conducted in the absence of any commercial or financial relationships that could be construed as a potential conflict of interest.

Copyright (C) 2019 Perng, Goodrich, Cardenas and Watkins. This is an open-access article distributed under the terms of the Creative Commons Attribution License (CC $B Y)$. The use, distribution or reproduction in other forums is permitted, provided the original author(s) and the copyright owner(s) are credited and that the original publication in this journal is cited, in accordance with accepted academic practice. No use, distribution or reproduction is permitted which does not comply with these terms. 